\title{
Effect of Use of Ambulance as Emergency Medical Service in Patients of ST-Elevation Myocardial Infarction \\ Majid Bashir ${ }^{1}$, Kubra Maryam ${ }^{1}$, Nazeer Ahmed ${ }^{1}$, Tariq Mehmood ${ }^{2}$, Adnan Aslam ${ }^{3}$
}

\section{ABSTRACT}

Objective: To study the frequency of ambulance utilization by the St-elevation Myocardial Infarction patients to reach hospital, perception of ambulance users about the facilities available in the ambulance, and evaluate the clinical outcomes of STEMI between ambulance users and non-users.

Study Design: Cross-section survey-based study.

Place and Duration of Study: The study was carried out in the Department of Cardiology at Chaudry Pervaiz Elahi Institute of Cardiology (CPEIC) Multan from $14^{\text {th }}$ April 2020 to $14^{\text {th }}$ September 2020.

Materials and Methods: Patients with the diagnosis of STEMI were included in the study and were classified into two groups' ambulance and non-ambulance users, to reach the facility. Patients' demographics, initial presenting symptoms, availability of ambulance, and time to reach the hospital were recorded. Moreover, they were followed for complication during their stay and base line laboratory indicators. Ambulance users were also evaluated for their perception about availability of medical services in the ambulance. The data collected from both of the groups were compared through student's t-test and chi-square test. Statistical value less than 0.05 was considered as significant.

Results: Out of 300 patients, $32.6 \%$ were ambulance users while $67.4 \%$ were non-ambulance user. No significant difference was found in age, gender, underlying comorbidity, and initial presenting symptoms between two groups. Majority of ambulance users (74\%) arrived in less than 45 minutes. Different complications were recorded but no significant difference was found between two groups. Majority of ambulance users $31.5 \%$ were neutral about the level of satisfaction for ambulance facilities.

Conclusion: Frequency of ambulance utilization by STEMI patients is not only low in Pakistan, but ambulance system is also not successful in producing significant change in clinical outcomes. Therefore an awareness campaign along with ambulance improvement campaigns should be launched to bring a meaningful change.

Key Words: Ambulance System, Emergency Services, STEMI.

How to cite this: Bashir M, Maryam K, Ahmed N, Mehmood T, Aslam A. Effect of Use of Ambulance as Emergency Medical Service in Patients of ST-Elevation Myocardial Infarction. Life and Science. 2021;2(3): 107-110. doi: http://doi.org/10.37185/LnS.1.1.189

This is an Open Access article distributed under the terms of the Creative Commons Attribution License (http://creativecommons.org/licenses/by/4.0), which permits unrestricted use, distribution, and reproduction in any medium, provided the original work is properly cited.

\section{Introduction}

Cardiovascular disease (CVD) is most frequent cause

${ }^{1}$ Department of Cardiology

Nishtar Medical University and Hospital, Multan

${ }^{2}$ Department of Cardiology

Ch. Pervaiz Elahi Institute of Cardiology (CPEIC), Multan

${ }^{3}$ Department of Statical Analysis

Bahauddin Zakariya University, Multan

Correspondence:

Dr. Adnan Aslam

Department of Statical Analysis

Bahauddin Zakariya University, Multan

E-mail: adnanaslam628@yahoo.com

Funding Source: NIL; Conflict of Interest: NIL

Received: Mar 17, 2020; Revised: May 28, 2021

Accepted: Jun 21, 2021

107 of mortality according to World Health Organisation. 17.5 million died in 2013 because of this disease, which equals 31 percent of total global mortality rate. It is predicted CVD will remain the prime cause of mortality until 2030. ${ }^{1}$ Although CVD has affected the entire world yet the Asian region has been witnessing higher CVD burden in contrast to western world. Pakistan, being a developing country, has also been burdened by the growing prevalence of disease. $^{2}$

Among CVD, acute St elevation myocardial infarction (STEMI) is most lethal and accounts for 15 to 20 percent deaths. ${ }^{3}$ The developed world has been practicing early detection approaches to improve the 
clinical outcomes of the disease., Early Electro Cardio Graph (ECG) and detection of STEMI decreases associated complications, infarct size and better reperfusion times. ${ }^{4}$

According to World Health Organisation, emergency medical services are critical in improving the clinical outcomes of multiple diseases including CVD. ${ }^{5}$ Ambulance is a pre hospital emergency system which serve as first point of contact between the patient and healthcare system. In the modern world it is equipped with latest diagnostic and therapeutic resources that help in reducing the mortality rate. ${ }^{6,7}$ However, in Pakistan no established data has been found regarding the frequency of use of ambulance and its clinical outcomes among the suspected cases of STEMI. This study was designed to ascertain the practice of use of ambulance among the cardiac patients of Pakistan. Moreover, the perception of people about pre hospital facilities in the ambulance were evaluated and their effect on the clinical outcome of the disease was determined.

\section{Materials and Methods}

A cross-sectional survey-based study was conducted in the cardiac wards of Chaudry Pervaiz Elahi Institute of Cardiology (CPEIC) Multan for the period of six months from $14^{\text {th }}$ April 2020 to $14^{\text {th }}$ September 2020. Patients who were admitted in the cardiac centres were included in study through random sampling technique after confirmation of occurrence of STEMI from the physician. A sample size of 300 was calculated with $80 \%$ value of power set and $p$ value of less than 0.05 significance. Patients from any age group male and female were included in the study. Patients were informed of the study objectives and their inclusion in the study was confirmed after getting their consent. Patients were divided into two groups: ambulance and non-ambulance users. Nonambulance group comprised of patients who used private transportation to reach at hospital. The study was conducted after approval from hospital ethical board. A standardized questionnaire was prepared to collect data about the clinical outcomes of use of ambulance. Confirmed cases of STEMI were then questioned for the mode of transportation to hospital on the appearance of various disease symptoms including chest pain. Patients' case files were used to collect medical information and their in-hospital condition was observed. Collected data comprised of patients' demographics, time taken to reach the hospital, emergency severity index (ESI) score, ranging between 1 to 5 on the scale from lowest to highest severity of disease, diagnostic reports, in-hospital complications, STEMI treatment approach, availability of ambulance services, and extent of satisfaction with the pre-hospital services in the ambulance. Perception of ambulance users were graded from very unsatisfied to very satisfied. Patients who died after the initial treatment or who refused to stay in the hospital against the medical advice were excluded from the study.

\section{Data Analysis}

SPSS version 25.0 was used for statistical analyses. Categorical data was represented in the form of frequencies with $95 \%$ confidence interval $(\mathrm{Cl})$ while continuous variables were presented as mean with $95 \% \mathrm{Cl}$. The continuous variables between the two groups were compared through student's t-test while the Pearson's chi-squared test was used for the comparison of categorical data to test the level of significance. Statistical value less than 0.05 was considered as statistically statistical.

\section{Results}

A total of 300 participants were included in the study out of which $98(32.6 \%)$ arrived through ambulance while 202 (67.3\%) used alternative means. No significant difference was found between mean of ages of two groups (0.84) Majority of the patients were males 210 (70.3\%), More than half, 55 patients (55.6\%) among ambulance users and 105 (52.3\%) of non-ambulance users, had hypertension as an underlying comorbidity; however, there was no significant difference between the studied groups in terms of underlying diseases. Chest pain was the most common presenting symptom among the participants; The number of ambulance user who came with the presenting complaint of chest pain were 55 while non-ambulance users were 166 . Chest pain was followed by dyspnoea, diaphoresis, and shoulder pain in both the groups while the last two symptoms occurred without any significant difference. When non-ambulance users were asked about reason for using other transport, 21 (10\%) of them responded that they approached the ambulance facility, but the services were not available. Further, $73.8 \%$ of ambulance user took less than 45 minutes to reach hospital as compared to 
38.8\% of non-ambulance users (Table 2). Different complications including cardiogenic shock, atrial fibrillation, ventricular tachycardia, ventricular fibrillation, transient ischemic attack, atrial flutter, myocardial infarction, and stent thrombosis were reported at different occurrence rates among patients of two groups. Majority of respondents 31 (31.5\%) were neutral in their response while 2 (2.1\%) were not satisfied at all (Table 3).

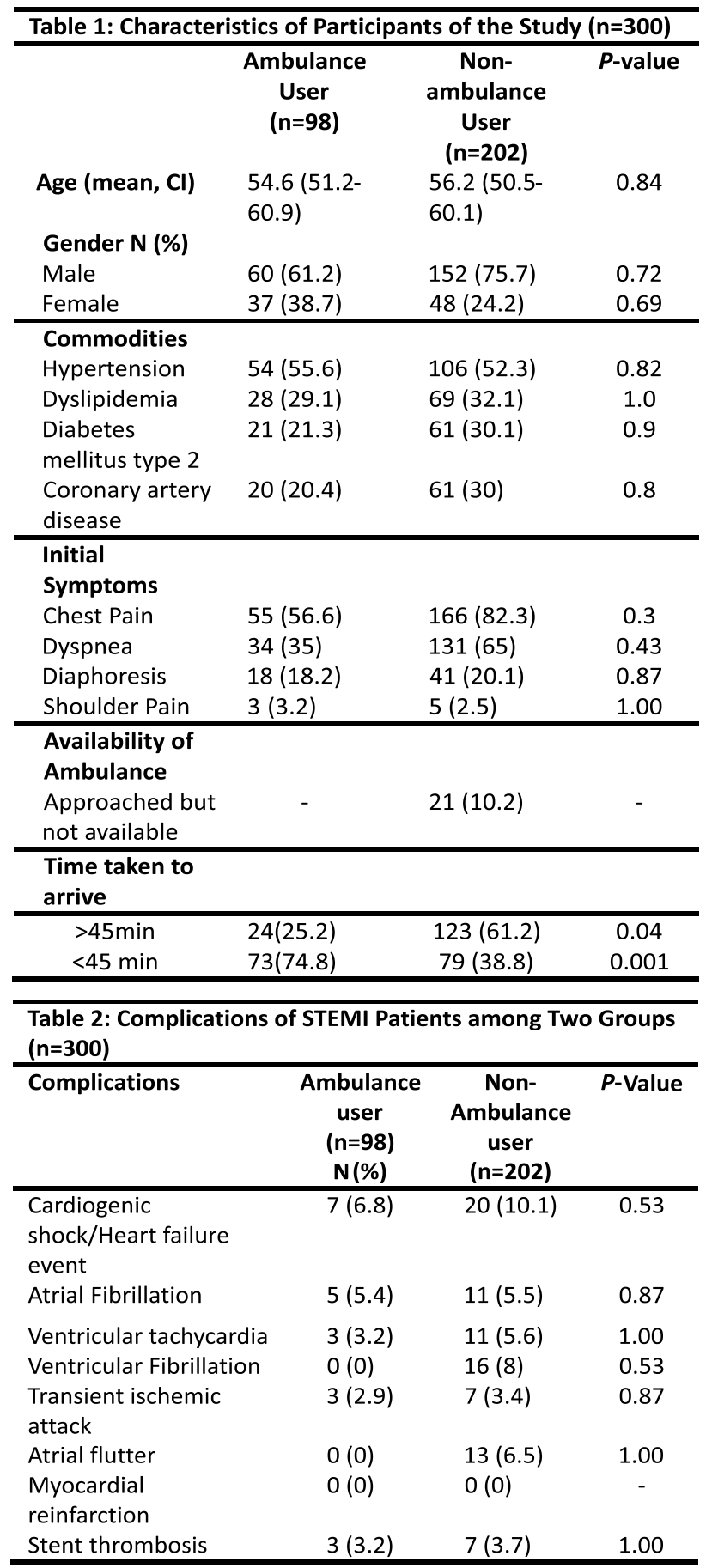

109

\begin{tabular}{lllllll}
\hline Table 3: Perception of Ambulance Users about its Facilities & & \\
\hline How & Very & Somewhat & Neutral & Somewhat & Very & P-value \\
satisfied & Unsatisfied & Unsatisfied & & Satisfied & Satisfied & \\
are you & $2(2.1)$ & $23(23.5)$ & $31(31.5)$ & $25(25.3)$ & 17 & 0.05 \\
with the & & & & & $(17.6)$ & \\
current & & & & & & \\
ambulance & & & & & & \\
services & & & & & \\
level of & & & & & \\
care given & & & & & & \\
to ST- & & & & & & \\
Elevation & & & & & & \\
patients? & & & & & & \\
N(\%) & & & & & & \\
\hline
\end{tabular}

\section{Discussion}

Pre-hospital emergency treatment plays a significant role in limiting the mortality of people suffering from serious illnesses. Since, high prevalence of cardiovascular disease (CVD) has been reported in Pakistan, this study was conducted to evaluate the impact of pre-hospital services in the form of ambulance in Pakistan on clinical outcome of STEMI. It was also envisaged to determine the general trend toward the utilization of such service and perception of people about its effectiveness.

It was found only $32.6 \%$ of patients utilized the ambulance facility to reach the hospital. In a similar previous study conducted, the rate was as low as $4 \% ;^{6}$ In the developed world, rate of use of ambulance is significantly high ${ }^{8,9}$ In our study among the non-user of ambulance, $10 \%$ claimed that they tried to access the service but the either it was nonresponding or failed to reach on time. One of the other reasons might be lack of knowledge among the general public about the gravity of situation. It was observed that majority of non-user had chest pain as the initial presenting symptom which reflected that patients were unaware of possible association of chest pain with STEMI. Previous studies have found that people who had awareness about STEMI were inclined to use ambulance as means of transportation to hospital. ${ }^{10}$

In this study, no significant difference was found between basic characteristics such as age, demographics and EMI score between two observed groups. However, on exploration of past medical history it was found that patients with past experience of any cardiovascular disorder or other serious illness were more likely to use ambulance and was confident of pre-hospital services. These results were in compliance with recent studies of conducted on emergency system of resourceful nations such as UAE, Oman, and Saudia Arabia. ${ }^{11,12}$ 
In contrast to ambulance system of modern world where pre-hospital treatment assist in reducing complications of the disease, unfortunately in our study no significant difference was found between prognosis of patients from two groups and frequency of complication were almost similar. This reflects the ineffective condition of pre-hospital facilities in Pakistan. Similarly, the ambulance might lack in training to deal with emergency situation. In an early study, similar results were shown where death rate was high among the patients who were reaching through ambulance. Since the study covered the ambulance system of six main cities of Pakistan, higher death rate even among ambulance users predict the flawed emergency system of Pakistan. ${ }^{6}$ Majority of respondents were neutral in their opinion and were neither satisfied nor unsatisfied which goes in compliance with previous studies. ${ }^{13,14,15}$ Our study, however, lacks on several fronts. Firstly, the selected sample size was very small and no demographic variation was considered. The treatment protocol were variable with no quality assurance on the emergency standards. The effectiveness of results can be improved by overcoming the shortcomings of the study.

\section{Conclusion}

Despite the fact that modern world is actively using pre-hospital emergency services to halt the complications of STEMI, frequency of ambulance utilization is not only low in Pakistan but ambulance services are so far ineffective in improving the clinical outcomes.

\section{REFERENCES}

1. Callachan EL, Alsheikh-Ali AA, Bruijns S, Wallis LA. Physician perceptions and recommendations about pre-hospital emergency medical services for patients with ST-elevation acute myocardial infarction in Abu Dhabi. Journal of the Saudi Heart Association. 2016; 28: 7-14.

2. Liaquat $A$, Javed $Q$. current trends of cardiovascular risk determinants in Pakistan. Cureus. 2018; 10: e3409.

3. Canto JG, Rogers WJ, Chandra NC, French WJ, Barron HV, Frederick PD, et al. The association of sex and payer status on management and subsequent survival in acute myocardial infarction. Archives of internal medicine. 2002; 162: 587-93.

4. Rebeiz A, Sasso R, Bachir R, Mneimneh Z, Jabbour R, EI Sayed M. Emergency Medical Services Utilization and
Outcomes of Patients with ST-Elevation Myocardial Infarction in Lebanon. The Journal of Emergency Medicine. 2018; 55: 827-35.

5. Kobusingye OC, Hyder AA, Bishai D, Hicks ER, Mock C, Joshipura M. Emergency medical systems in low-and middle-income countries: recommendations for action. Bulletin of the World Health Organization. 2005; 83: 62631.

6. Zia N, Shahzad H, Baqir SM, Shaukat S, Ahmad H, Robinson $C$, et al. Ambulance use in Pakistan: an analysis of surveillance data from emergency departments in Pakistan. BMC Emergency Medicine. 2015; 15: S9.

7. Snooks HA, Anthony R, Chatters R, Dale J, Fothergill R, Gaze $S$, et al. Support and Assessment for Fall Emergency Referrals (SAFER) 2: a cluster randomised trial and systematic review of clinical effectiveness and costeffectiveness of new protocols for emergency ambulance paramedics to assess older people following a fall with referral to community-based care when appropriate. Health technology assessment. 2017; 21: 1-218.

8. Lindskou TA, Mikkelsen S, Christensen EF, Hansen PA, Jørgensen G, Hendriksen OM, et al. The Danish prehospital emergency healthcare system and research possibilities. Scandinavian journal of trauma, resuscitation and emergency medicine. 2019; 27: 1-7.

9. Kobayashi A, Misumida N, Aoi S, Steinberg E, Kearney K, Fox JT, et al. STEMI notification by EMS predicts shorter door-toballoon time and smaller infarct size. The American Journal of Emergency Medicine. 2016; 34: 1610-3.

10. Bray JE, Stub D, Ngu P, Cartledge S, Straney L, Stewart M, et al. Mass Media Campaigns Influence on Prehospital Behavior for Acute Coronary Syndromes: An Evaluation of the Australian Heart Foundation's Warning Signs Campaign. Journal of the American Heart Association. 2015; 4: e001927.

11. AlHabib KF, Sulaiman K, Al Suwaidi J, Almahmeed W, Alsheikh-Ali AA, Amin H, et al. Patient and System-Related Delays of Emergency Medical Services Use in Acute STElevation Myocardial Infarction: Results from the Third Gulf Registry of Acute Coronary Events (Gulf RACE-3Ps). PLOS ONE. 2016; 11: e0147385.

12. Callachan EL, Alsheikh-Ali AA, Nair SC, Bruijns S, Wallis LA. Outcomes by mode of transport of ST elevation MI patients in the United Arab Emirates. Western Journal of Emergency Medicine. 2017; 18: 349.

13. Chandran A, Ejaz K, Karani R, Baqir M, Razzak J, Hyder AA. Insights on the effects of patient perceptions and awareness on ambulance usage in Karachi, Pakistan. Emergency Medicine Journal. 2014; 31: 990-3.

14. Phillips JS. Paramedics' perceptions and experiences of NHS 111 in the south west of England. Journal of Paramedic Practice. 2020; 12: 227-34.

15. Lowthian JA, Cameron PA, Stoelwinder JU, Curtis A, Currell $A$, Cooke MW, et al. Increasing utilisation of emergency ambulances. Australian Health Review. 2011; 35: 63-9. 